\title{
Influence of the Length and Position of the Collision Cell on the Resolving Power Attainable from Collisionally Activated Processes in Sector Instruments
}

\author{
C. Leather, A. C. R. Wilkins, and A. G. Brenton \\ Mass Spectrometry Research Unit, Swansea University Wales, Swansea, United Kingdom
}

\begin{abstract}
The energy resolution in a high resolution tandem mass spectrometer was investigated in terms of the length and position of the central collision cell. Both these parameters have to be chosen carefully to eliminate collisional broadening phenomena that can severely limit the energy resolution in such instruments. A theory that describes collisional broadening, to first order, has been derived that accounts for the observed phenomena. It was found experimentally that collisional broadening can be reduced to less than $0.001 \mathrm{eV}$ per kiloelectronvolt collision energy. Experimental data confirmed that only the size of the resolving slits and thermal broadening, due to the target gas, limited the ultimate energy resolution available. (J Am Soc Mass Spectrom 1995, 6, 212-219)
\end{abstract}

$\mathrm{T}$ The versatility of the mass spectrometer has long been recognized and described as the "complete laboratory" when it is configured as a tandem mass spectrometer. Studies of collision-induced processes have featured prominently in the development of mass spectrometry both at the fundamental [1] and the applied [2] levels. Sector instruments have been employed widely, particularly when the energetics of a reaction were to be studied, both for dissociative and nondissociative processes [3]. Early tandem mass spectrometry studies concentrated on dissociative reactions in double-focusing instruments, initially of conventional geometry with high voltage [4] or linked scan [5] techniques, but later on reversed-geometry instruments using mass-analyzed ion kinetic energy spectrometry (MIKES) [6]. For high voltage and MIKE scans the resolution obtained was poor because kinetic energy release broadened the peak shape, whereas in the case of a B/E-linked scan, these effects could be reduced significantly. Physical chemists, however, had taken advantage of these effects, for example the detailed study of a peak shape that arises in a MIKE spectrum can reveal the intimate energetics of a fragmentation process particularly when the peak contains structure [7]. In this latter case the energy and angular resolution that could be achieved with the instrument were critical factors in determination of an accurate kinetic energy release distribution from which the en-

Address correspondence to Gareth Brenton, Mass Spectrometry Research Unit, Swansea University Wales, Swansea SA2 8PP, UK. ergetics of the fragmentation mechanism could be gleaned. Only in a limited number of such studies was the effect of the size and position of the collision cell on the recorded spectrum considered in detail [8]. So far we have considered only reactions in which the ion undergoes fragmentation, whereas the results given in this article will be concerned only with nondissociative processes; that is, translational energy loss (or gain) spectra where the peak shape of the selected ion beam is kept as narrow as possible so as to obtain high resolving power. However, our general findings may be applied to dissociative processes.

Translational energy spectroscopy (TES) is a technique in which a kiloelectronvolt ion beam in a mass (or energy) spectrometer is allowed to collide with a neutral target gas. The ion can undergo excitation (or deexcitation), whereas the target can be excited only. The energy to drive these excitation processes can be derived only from the translational energy of the fast ion beam, and thus by measuring its energy loss (gain) spectrum one can measure directly the electronic and, where possible, the vibrational bands of the ionic and target species. To successfully conduct a TES measurement it is essential that the instrument be operated at high energy resolution, typically $0.1-\mathrm{eV}$ energy spread on the main unscattered ion beam, which for a translational energy of $3000 \mathrm{eV}$ corresponds to an energy resolution of $3 \times 10^{4}$. However, the peaks in the energy loss spectrum will be broader than the main beam. This loss in resolution is attributable to two main factors: thermal broadening $\left(\delta E_{t h}\right)$ and collisional 
broadening $\left(\delta E_{\mathrm{cb}}\right)$. If the energy loss peak width is $\delta E$, then

$$
\delta E=\sqrt{\left(\delta E_{\mathrm{mb}}\right)^{2}+\left(\delta E_{\mathrm{th}}\right)^{2}+\left(\delta E_{\mathrm{cb}}\right)^{2}}
$$

where $\delta E_{\mathrm{mb}}$ is the width of the main beam of ions at zero energy loss. These widths are referred to at full width at half-maximum (FWHM). The thermal broadening component has been derived elsewhere [9] and is related to the energy loss $(\Delta E)$ by

$$
\delta E_{\mathrm{th}}=4 \ln 2 \frac{m_{1}}{m_{0}} \frac{k T}{E^{0}} \Delta E
$$

where $m_{1}$ is the mass of the ion, $m_{0}$ is the mass of the thermal target gas, $E_{0}$ is the collision energy, $T$ is the gas temperature, and $k$ is the Boltzmann constant. In a TES experiment it is desirable to limit the magnitude of both these broadening terms. To reduce $\delta E_{\text {th }}$, a suitable choice of heavy target gas and/or cryogenic cooling of the target is required. The collision broadening component, $\delta E_{\mathrm{cb}}$, can be defined in terms of three parameters: fractional translational energy loss $\left(\Delta E / E^{0}\right)$, the scattering angle $(\theta)$ in the plane of dis- persion, and the scattering angle ( $\rho$ ) orthogonal to that plane. This relationship can be represented in terms of a power series to third order [10]. In this article we are concerned primarily with determination of the main collisional broadening component that is due to firstorder angular scattering. This effect can be appreciated by reference to Figure 1a, which shows scattering of an initially collimated main beam of ions at three positions within a long collision cell. Scattering at the center of the cell (position $C$ ) leads to ideal conditions whereby the scattered ions will be refocused to a sharp image at $I$. Scattering away from the center at $C^{\prime}$ and $C^{\prime \prime}$ leads to refocusing off the final focal plane at $I^{\prime}$ and $I^{\prime \prime}$, respectively. In both these latter cases the recorded beam will be broadened with an associated loss of sensitivity. Scattering at $C^{\prime}$ contributes more to collisional broadening than $C^{\prime \prime}$ under the assumption that the center of the collision cell is positioned on the central focal plane (refer to Figure 2). However, this cannot be guaranteed, particularly in our apparatus, because there is a $Y$ focus lens (YF1) employed before electric sector analyzer (ESA 1) that has the effect of varying this position. The present work describes the quantitation of first-order collisional broadening by
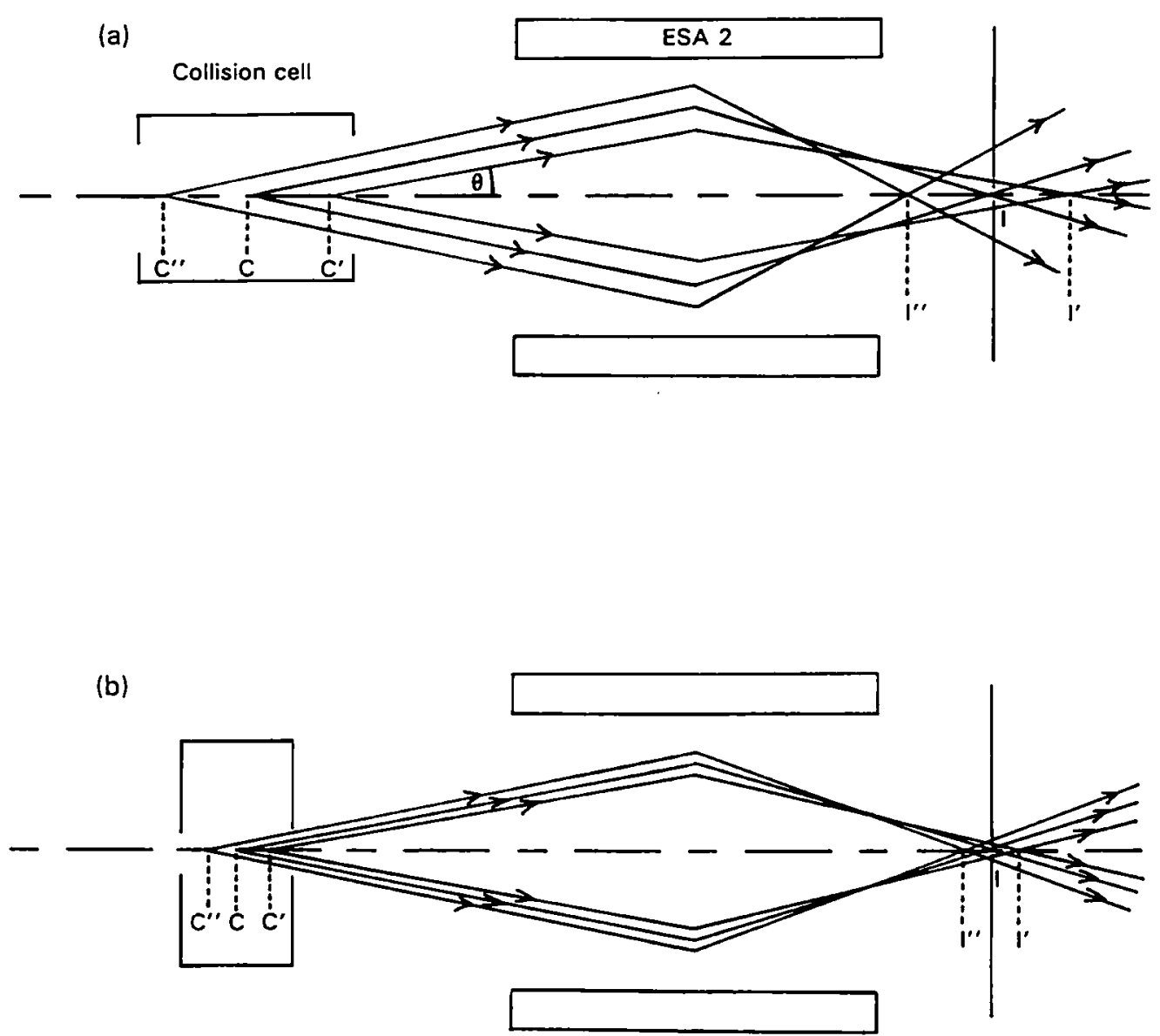

Figure 1. Schematic ion trajectory diagram that illustrates the effect of scattering at three positions $\left(C, C^{\prime}\right.$ and $C^{\prime \prime}$ ) in a collision on the final image: (a) normal length collision cell and (b) short path length collision cell. 


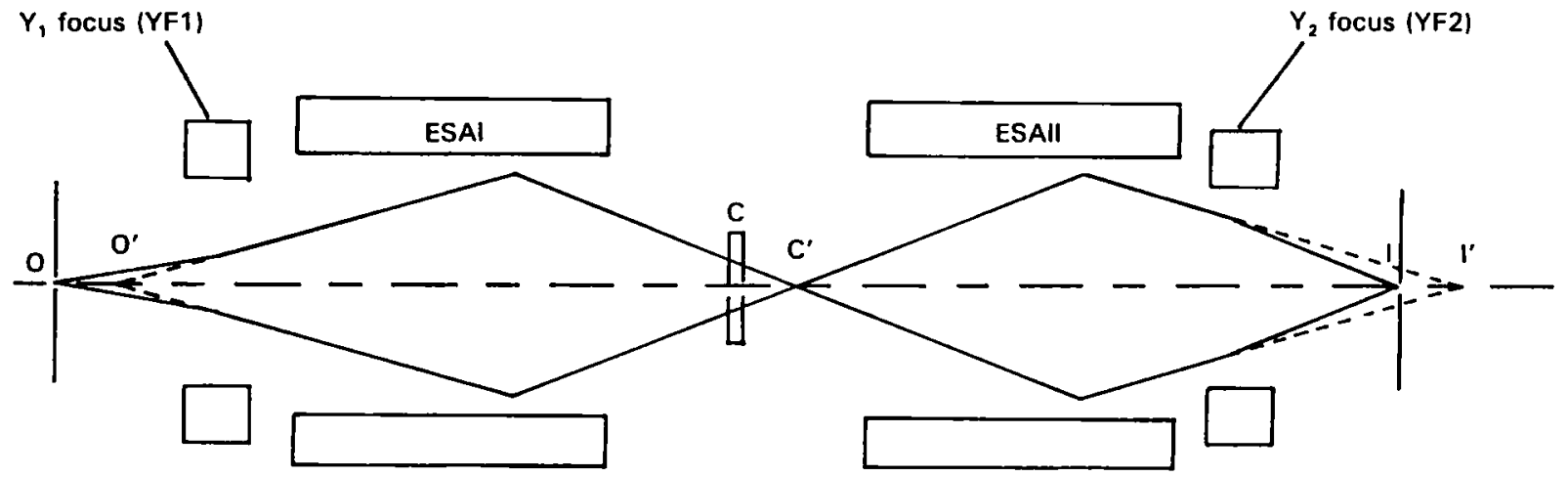

Figure 2. Focusing diagram for the translational energy loss spectrometer in the plane of dispersion illustrating the effects of the $Y$-focusing lenses $Y F 1$ and $Y F 2$. $O$, high resolution object slit; $l$, high resolution image slit; $C$, optical center between electrostatic analyzers; $C^{\prime}$, actual mid focal point.

translation of the collision cell along the $x$-axis, and a theoretical model for collisional broadening is derived in terms of instrumental ion optical parameters.

\section{Experimental}

Results were obtained by using a high-resolution translational energy spectrometer constructed in our laboratory and described in detail elsewhere [11]. Briefly, the instrument is based on a pair of symmetrically arranged identical $90^{\circ}$ electrostatic analyzers, which are shown in Figure 3. An electron impact source (electron energy $140 \mathrm{eV}$ ) used $\mathrm{N}_{2}$ gas to pro- duce $\mathrm{N}^{+}$ions that were accelerated to $3 \mathrm{keV}$ and mass selected by a 5 -in. radius magnet. The ion beam passed through ESA 1 and ESA 2, which together provide very high energy resolution for energy loss processes that occur in the collision cell. Figure 4 illustrates the design of the thin collision cell specifically employed for these studies. The $x$-axis was made as short as possible, $x_{\mathrm{c}}=2 \mathrm{~mm}$, to restrict the spatial region over which collisions, and thus beam scattering, occurs. The width $\left(y_{c}\right)$ of the entry and exit apertures of the cell were reduced $\left(y_{c}=0.3 \mathrm{~mm}\right)$ to give a very low gas conductance $\left(0.163 \mathrm{~L} \mathrm{~s}^{-1}\right)$. In conjunction with a $700-L$ $\mathrm{s}^{-1}$ diffusion pump located directly beneath the cell,

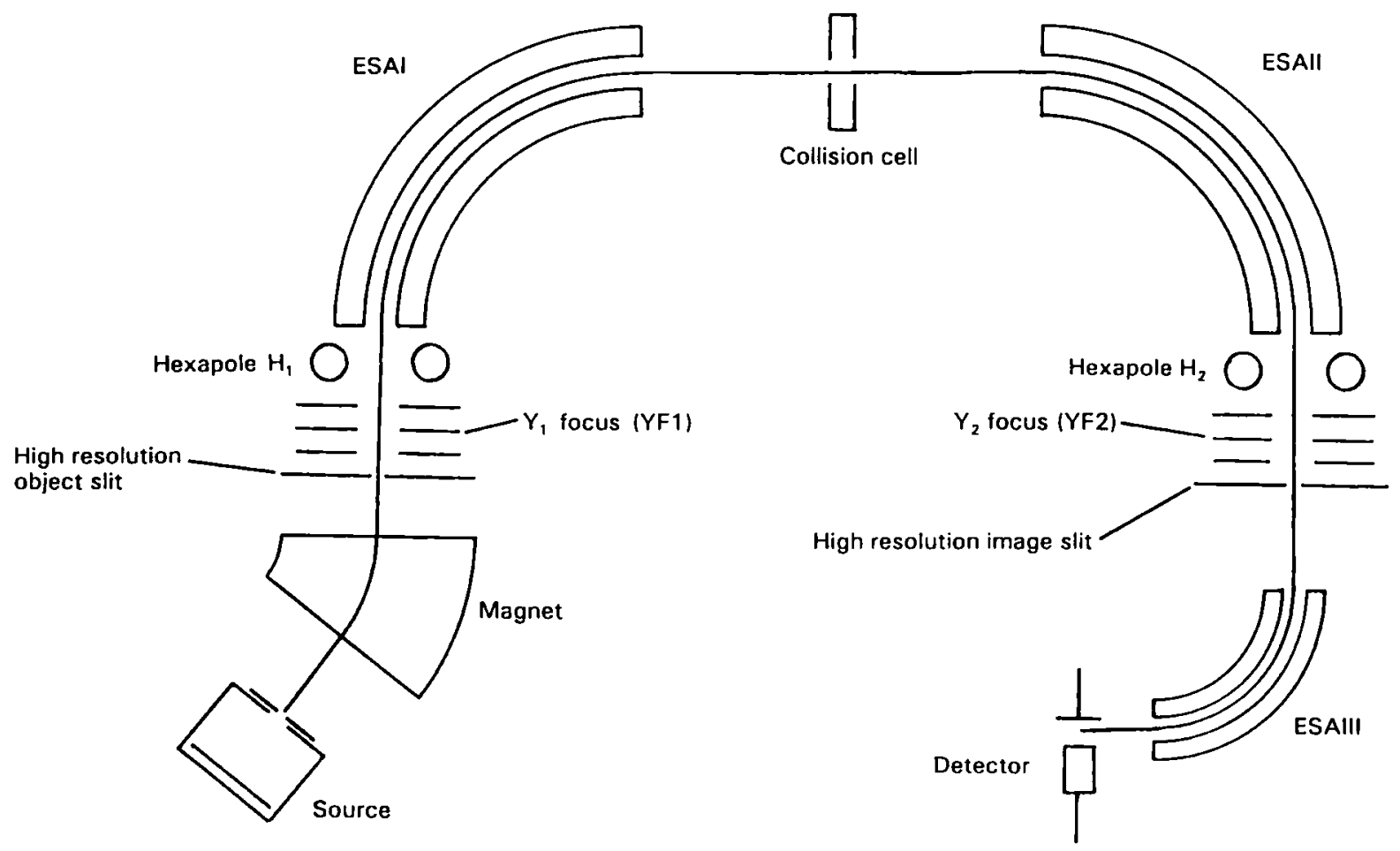

Figure 3. Schematic diagram of the translational energy spectrometer at Swansea, illustrating the positions of the $Y$ focusing lenses and hexapoles. 

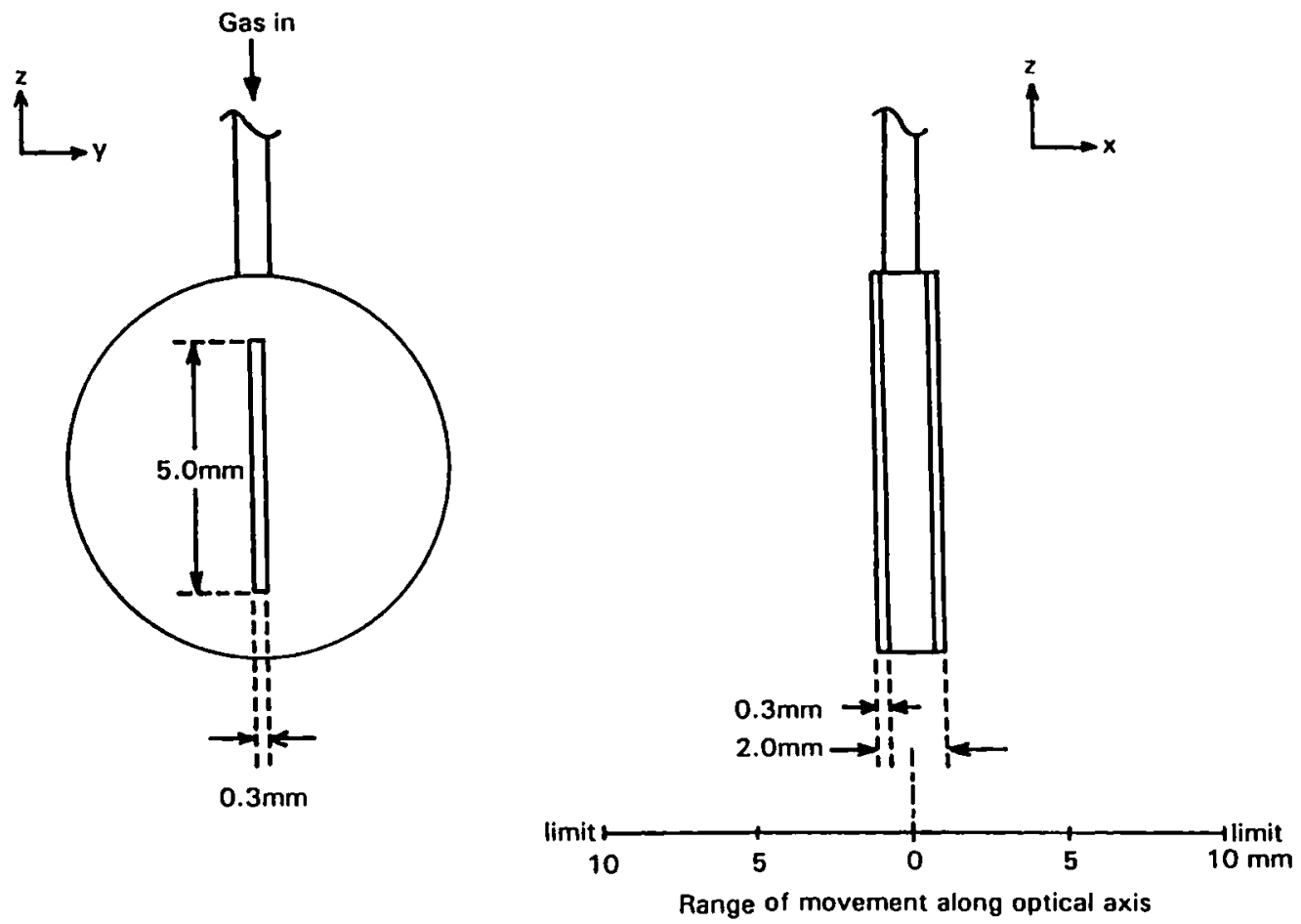

Figure 4. Diagram of the short path length collision cell used in this investigation.

this achieved a differential pressure between the interior of the cell and the surrounding vacuum housing of $\sim 1600: 1$. Thus it could be ensured that the number of collisions that occurred outside the cell was very small and that a near-constant target gas thickness $\pi$ was produced, compared to the longer path length cell $\left(x_{\mathrm{c}}=10 \mathrm{~mm}, y_{\mathrm{c}}=1 \mathrm{~mm}\right)$ we previously employed, where

$$
\pi=x_{\mathrm{c}} P_{\mathrm{cc}}
$$

and $P_{c c}$ is the average pressure within the cell. A reduction in the length of the collision cell leads to reduced collisional broadening (Figure 1) and thus gives a high ultimate resolving power. To quantitate the effects of first-order collisional scattering, the experiment we designed was to translate the collision cell along the $x$-axis and measure the resultant change in energy resolution for an energy loss peak. The cell was mounted via a stainless steel bar to a high precision translator (Vacuum Generators Ltd., Hastings, U.K., model HPT1) that allowed translational motion along the cell's $x$-, $y$-, and $z$-axes $(25,25$, and $150 \mathrm{~mm}$, respectively), and rotation about the $z$-axis $\left(360^{\circ}\right)$.

The pressure within the cell $\left(P_{c c}\right)$ was estimated from the pressure indicated by the ion gauge $\left(P_{i}\right)$ located close to the cell and the conductance of the cell apertures. $P_{\mathrm{cc}}$ was calculated to be a factor $1.6 \times 10^{3}$ greater than $P_{\mathrm{i}}$ and

$$
P_{\mathrm{cc}}=1.6 \times 10^{3} S_{\mathrm{i}} P_{\mathrm{i}}
$$

where $S_{i}$ is the ion gauge sensitivity for the gas (for $\left.\mathrm{N}_{2}, S_{\mathrm{i}}=1\right)$. Collision cell pressures $\left(P_{\mathrm{cc}} \leq 2 \times 10^{-3}\right.$ torr for $\mathrm{N}_{2}$ ) were set so that single collision conditions in the cell were maintained.

The scattered ions were energy analyzed by the second electric sector (ESA 2) and passed through a high resolution image slit. A smaller ESA (radius $=2.5$ in.) was located after the image slit to eliminate any spurious signals generated by neutral species and ions reflected off the surface of the second electric sector and image slit.

Because the vacuum housing of the collision cell region restricted the translational motion of the cell along the $x$-axis to $25 \mathrm{~mm}$, studies of collisional broadening were made only over this range. Initial experiments indicated that both greater image intensity and resolution could be obtained by a very slight angular inclination of the cell about the $z$-axis of $\sim 0.016 \mathrm{rad}$, which may arise from a mechanical and/or an ion optical deviation. By moving the cell along the $y$-axis, perpendicular to the beam path, it was possible to measure the effective aperture of the cell presented to the ion beam. It was important to ensure that the beam width was less than that of the cell aperture, particularly because any resultant peak-side ripple (caused when the beam strikes the edge of the collision cell) would reduce the resolution attainable for subsequent experimentation.

\section{Results}

Two energy loss processes were used to investigate the dependence of collisional broadening on the position of scattering. These were chosen because both give 


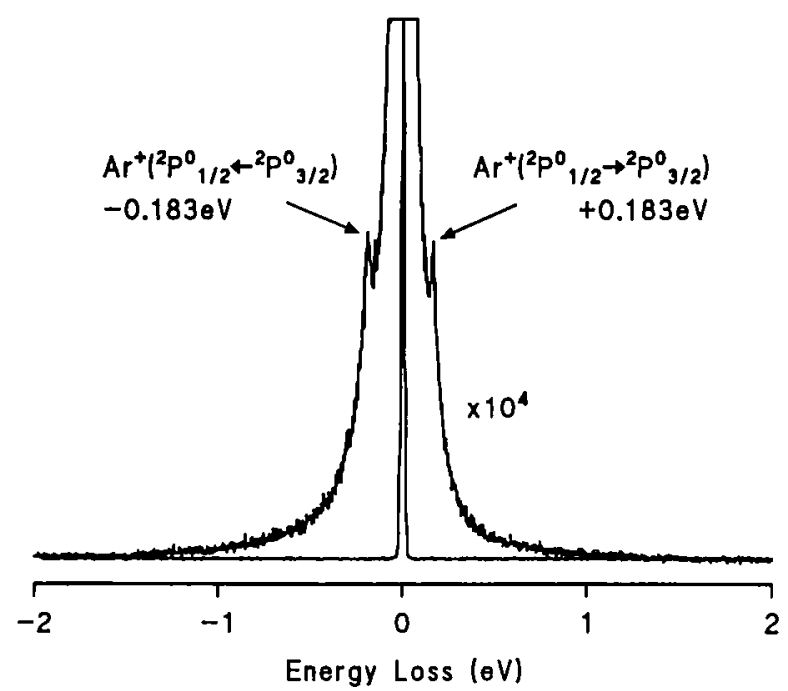

Figure 5. Translational energy loss spectrum of the spin-orbit splitting of the $3-\mathrm{keV} \mathrm{Ar}^{+}\left({ }^{2} P_{3 / 2,1 / 2}^{0}\right)$ collision with helium target gas that illustrates the inelastic transitions taken at a main beam energy resolution set to $0.06 \mathrm{eV}$ FWHM.

high resolution on the product beam and enable the collisional broadening component to be measured more precisely.

\section{$\mathrm{Ar}^{+}$in Collision with $\mathrm{He}$}

The spin-orbit splitting of $\operatorname{Ar}^{+}\left({ }^{2} P_{3 / 2,1 / 2}^{0}\right)$ is a demanding system to measure by TES and we thought it would be a useful first test. For this experiment a moderate energy resolution $(0.06 \mathrm{eV})$ was employed throughout. A typical spectrum produced from this system is given in Figure 5. The enlarged portion shows the inelastic $(-0.183-\mathrm{eV})$ and superelastic $(+0.183-\mathrm{eV})$ peaks. Figure 6 shows the variation in spectral resolution of these reactions as the collision cell moves away from its central (focused) position along the $x$-axis. It is clearly visible that the resolution falls off as the cell is displaced from the center $C$ (refer to Figure 2). Also, the collisional broadening effect is symmetrical about the center; that is, peaks $a$ and $g, b$ and $f, c$ and $e$, which are equally displaced from $c$, are very similar in product resolution. This system clearly shows the attested effect of collisional broadening in a double-focusing instrument.

\section{$\mathrm{N}^{+}$in Collision with $\mathrm{O}_{2}$}

Here, 3-keV N${ }^{+}$ions are incident upon $\mathrm{O}_{2}$ molecules. This system was chosen because it produces strong and well defined electronic transitions (refer to Figure 7a). The feature under scrutiny is the triplet-singlet molecular excitation transition $X^{3} \Sigma_{g}^{-} \rightarrow a^{1} \Delta_{g}$ on the inelastic energy loss side of the spectrum that appears at $1.90 \mathrm{eV}$. Again, peak width (FWHM) was monitored as a function of $x$-axis collision cell displacement over a total displacement of $18 \mathrm{~mm}$, between micrometer readings of 7 and $25 \mathrm{~mm}$ (Figure 8 ).

The instrument initially was tuned to a resolution of $0.06 \mathrm{eV}$ (FWHM) by using a standard method where the two $y$ focus controls (YF1 and YF2) were optimized, together with the alignment of the beam along the $z$-axis by using the rotation controls of hexapoles $\mathrm{H} 1$ and $\mathrm{H} 2$. This procedure was iterated until an optimum beam resolution was achieved, along with the maximum beam intensity, and was carried out without gas in the collision cell. For TES an instrument rarely is tuned to an actual energy loss peak because of limited sensitivity, which prevents optimization of peak shape in real time on an oscilloscope display. It has however been found that this procedure is effective for systems that have fixed collision cells and we adopted it here. Therefore the optimum position of the collision cell along the $x$-axis was unknown at the start and was to be determined.

Figure $7 \mathrm{~b}$ shows the energy loss peak for $\mathrm{N}^{+} / \mathrm{O}_{2}\left(X^{3} \Sigma_{\mathrm{g}}^{-} \rightarrow a^{1} \Delta_{\mathrm{g}}\right)$, which sits on the inelastic tail of the main beam at an energy loss of $1.90 \mathrm{eV}$. To obtain a more accurate measurement of the energy loss peak width, the baseline was subtracted (see Figure 7c). Figure 8a shows the change in measured peak width $(\delta E)$ as a function of the collision cell displace-
Figure 6. Variation of the $\operatorname{Ar}^{+}\left({ }^{2} P_{3 / 2,1 / 2}^{0}\right)$ spin-orbit TES spectrum as a function of collision cell position along the $x$-axis. Seven spectra were taken at positions of $0, \pm 2, \pm 4$, and $6 \mathrm{~mm}$ for the mid focal point $C^{\prime}$.

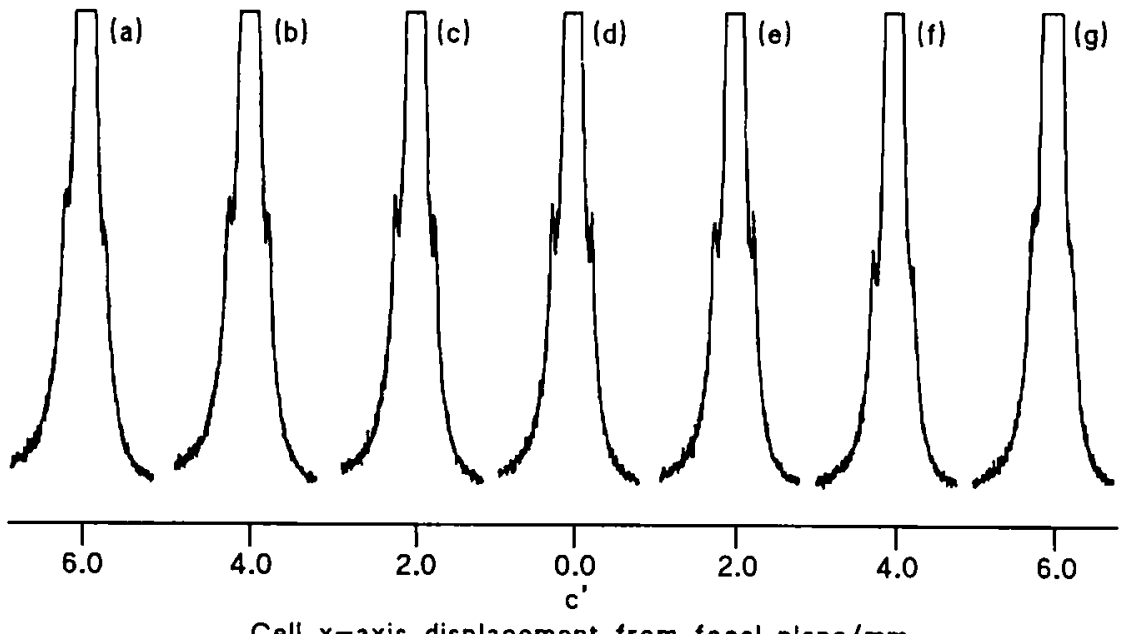

Cell $\mathrm{x}$-axis displacement from focal plane/mm 


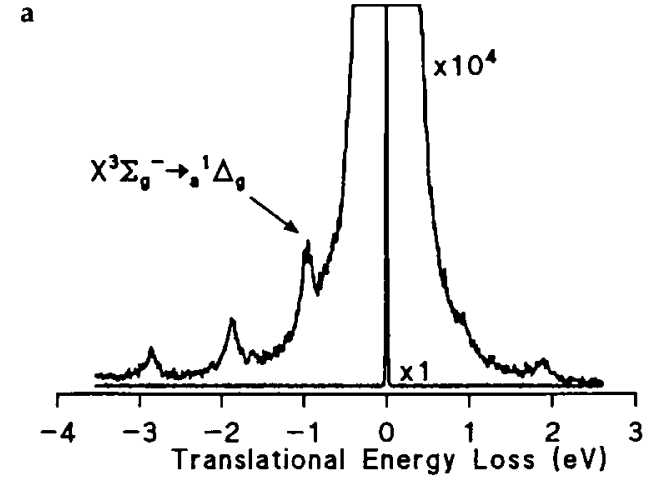

b
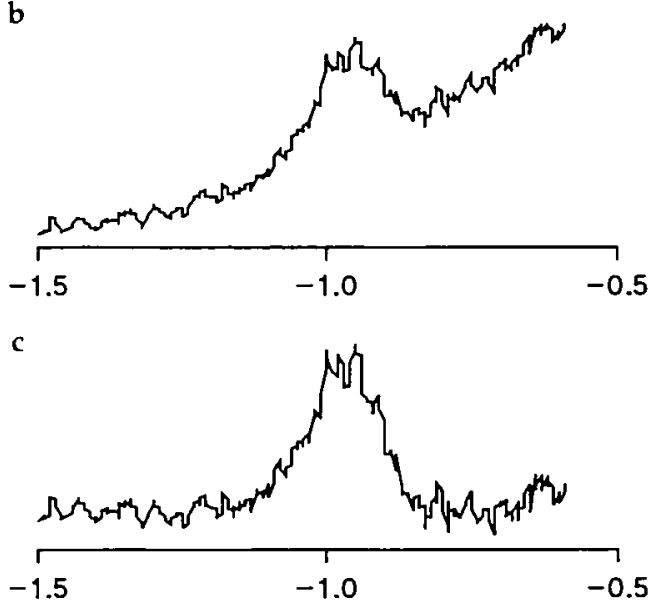

Figure 7. Translational energy loss spectrum of the $3-\mathrm{ke}^{\circ} \vee \mathrm{N}^{+}$ collision with $\mathrm{O}_{2}$; (a) Main features of the spectrum that show the electronic transition $\mathrm{N}^{+} / \mathrm{O}_{2}\left(X^{3} \Sigma_{k}^{-} \rightleftarrows a^{1} \Delta_{k}\right)$ at energy losses of $\pm 1.90 \mathrm{eV}$. (b) Enlargement of the energy loss peak from which (c) the effective width $(\delta E)$ of the collisionally broadened peak was measured by subtraction of the baseline.

ment. These measurements were repeated several times with the collision cell moved in opposite directions to ensure there were no asymmetric effects. It can be seen clearly that the peak width decreases linearly between 7 and $20 \mathrm{~mm}$, whereas it remains roughly constant above $20 \mathrm{~mm}$. There is some scatter of the data points over the linear region of the plot that is principally due to changes in the main beam width between individual runs; each run required $\sim 5 \mathrm{~min}$. A typical measurement sequence proceeded as follows: (1) Measure the main beam profile, (2) measure the energy loss peak, (3) recheck the main beam profile, and (4) check the accelerating voltage, which, in this experiment, was a critical parameter to maintain at a constant value. It was found that the main beam width, with gas in the cell, varied by up to $\pm 0.006 \mathrm{eV}$ throughout the course of the experiments. The scatter, seen in Figure $8 \mathrm{a}$, is partially due to this effect. More linear sets of data were obtained from one of the repeat runs, but the data presented here are more representative of all the data sets acquired.

The peak width, with the main beam width deconvoluted (by using eq 1) is shown in Figure 8c, and the

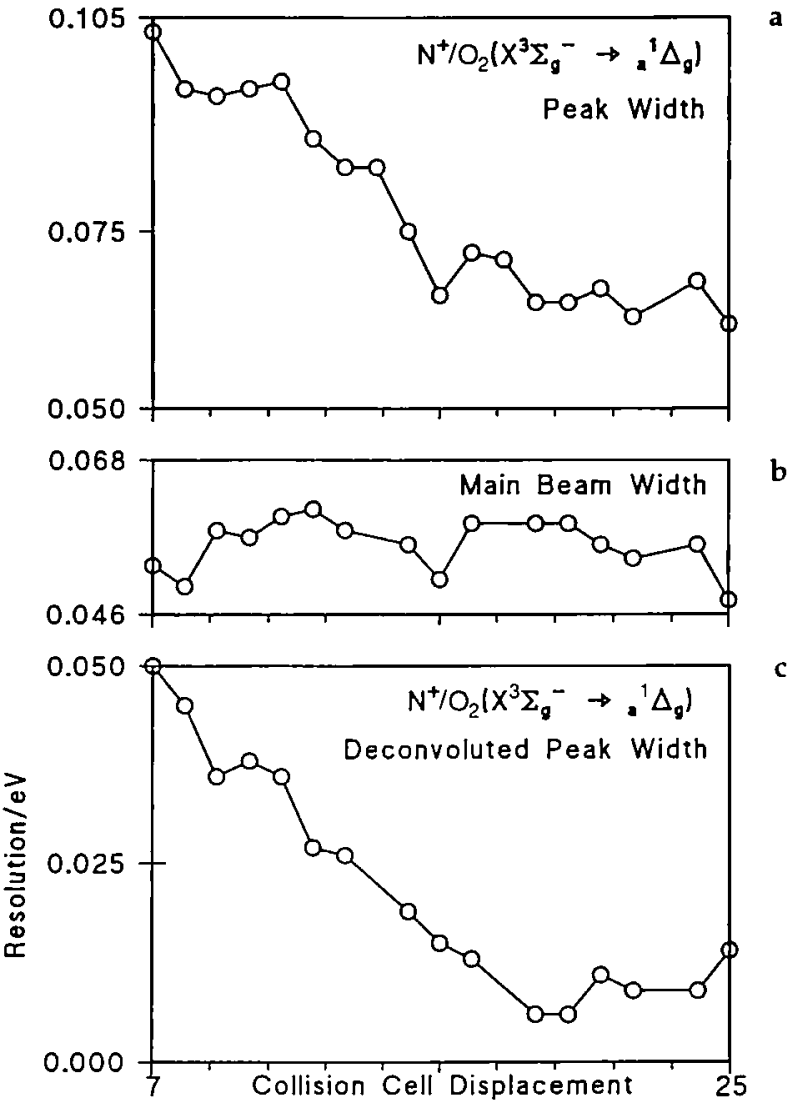

Figure 8. Plots of peak width variation in electronvolts measured at FWHM as a function of cell displacement for the collision of 3-keV N $\mathrm{N}^{+} / \mathrm{O}_{2}\left(X^{3} \Sigma_{k}^{-} \rightleftarrows a^{1} \Delta_{k}\right)$ (a) Actual width of the measured peak $(\delta E),(\mathrm{b})$ the width of the main beam of ions at that collision cell position, and (c) the peak width with the main beam $\left(\delta E_{\mathrm{mb}}\right)$ deconvoluted.

change in peak width now can be seen to follow a linear relationship between 7 and $20 \mathrm{~mm}$. The effect is simply first-order collisional broadening due to scattering at points away from the focal plane. It would be expected that at above approximately $20 \mathrm{~mm}$ the peak width would increase again given that the $20 \mathrm{~mm}$ position corresponds to the ideal focusing point. However, the $x-1 y-z$ translator was able to move only a further $5 \mathrm{~mm}$ and only a slight increase could be observed. This plateau is due to the finite target thickness, which will be wider than the cell itself $(2 \mathrm{~mm})$. In addition there will be contributions from the depth of the focal plane itself.

The minimum collision peak width $\left(\delta E_{\min }\right)$ is now very close to zero and is mainly because of thermal broadening $\left(\delta E_{\min }=0.006 \mathrm{eV}\right)$, which demonstrates that the instrument is operating at an effective energy resolution of $\left(V_{\mathrm{acc}} / \delta E_{\min }=3000 / 0.006=5 \times 10^{5}\right)$. For circumstances where the thermal broadening is considerably larger, for example $\mathrm{Ar}^{2+} / \mathrm{He}$, the ultimate resolution of the energy loss peaks will be reduced significantly and the only recourse to remedy this would be to cool the target gas or to use a molecular beam. 


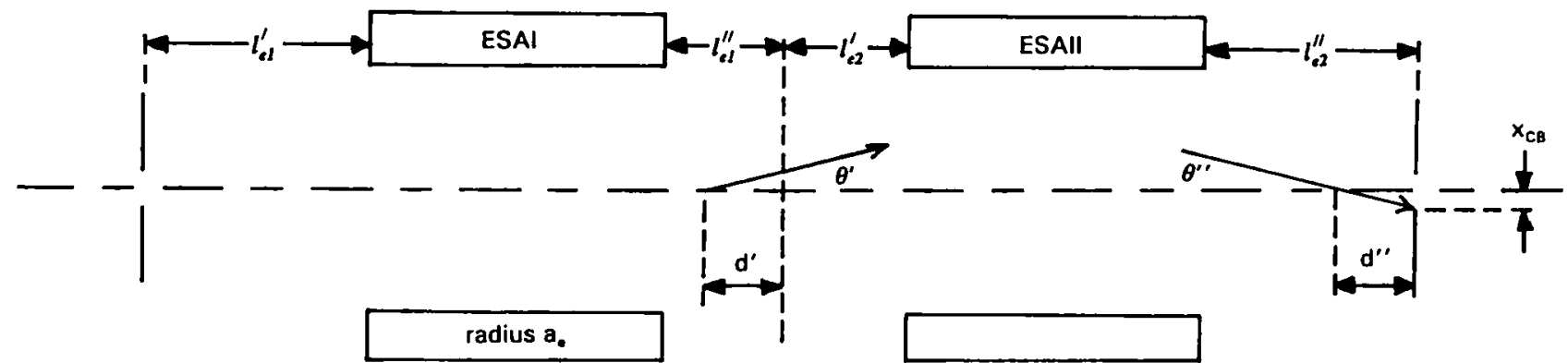

Figure 9. Ion optical parameters for the twin electric sectors ESA 1 and ESA 2. The object and image distances of ESA 1 and ESA 2 are $l_{01}^{\prime}, l_{01}^{\prime \prime}, l_{02}^{\prime}$, and $l_{02}^{\prime \prime}$, respectively. A collision is assumed to occur on the optical axis at $d^{\prime}$, from the mid focal plane, through a scattering angle of $\theta^{\prime}$, which intercepts the median plane at $d^{\prime \prime}$ at an angle of $\theta^{\prime \prime}$. The ion causes an aberration of $x_{c b}$ on the focal plane.

The collisional broadening $\left(\delta E_{\mathrm{cb}}\right)$ can be calculated readily and is given (in electronvolts) by

$$
\delta E_{\mathrm{cb}}=\frac{x_{\mathrm{cb}} E^{0}}{A_{\delta}}
$$

where $x_{\mathrm{cb}}$ is the image broadening (refer to Figure 9), $E^{0}$ is the collision energy, and $A_{\delta}$ is the energy dispersion.

Now, from standard ion optics [12],

$$
A_{\delta}=\frac{a_{\mathrm{e}}}{2}\left(1+\frac{f_{\mathrm{e}}}{l_{\mathrm{e} 2}^{\prime}-g_{\mathrm{e}}}\right)
$$

where

$$
f_{\mathrm{e}}=\frac{r_{\mathrm{e}}}{\sqrt{2} \sin \left(\sqrt{2} \phi_{\mathrm{e}}\right)}
$$

and

$$
g_{\mathrm{e}}=f_{\mathrm{e}} \cos \left(\sqrt{2} \phi_{\mathrm{e}}\right)
$$

where $a_{\mathrm{e}}$ is the mid-radius of the ESA and $\phi_{\mathrm{e}}$ is its deflection angle. Also

$$
x_{\mathrm{cb}}=d^{\prime \prime} \theta^{\prime \prime}=d^{\prime \prime} \frac{\theta^{\prime \prime}}{G_{\mathrm{e}}}
$$

where $\theta^{\prime}$ is the initial scattering angle, $\theta^{\prime \prime}$ is the angle that the ray makes on the final focal plane, and $G_{e}$ is the magnification of ESA 2, given by

$$
G_{e}=-\frac{f_{e}}{l_{\mathrm{e} 2}^{\prime}-g_{e}}
$$

From the lens equation for a cylindrical ESA [12],

$$
\left(\left(l_{\mathrm{e} 2}^{\prime}+d^{\prime}\right)-g_{\mathrm{e}}\right)\left(\left(l_{\mathrm{e} 2}^{\prime \prime}+d^{\prime}\right)-g_{\mathrm{e}}\right)=\left(f_{\mathrm{e}}\right)^{2}
$$

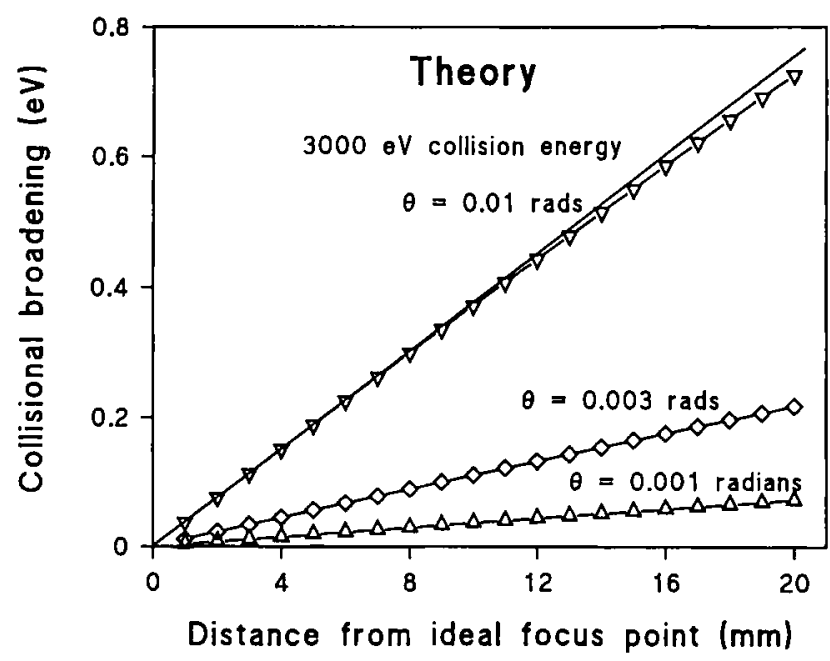

Figure 10. Theoretical prediction of the collisional broadening $\delta E_{\mathrm{cb}}$ for scattering angles of $\theta^{\prime}=0.001,0.003$, and 0.01 as a function of $d^{\prime}$ at $3-\mathrm{keV}$ collision energy.

Rearranging eq 11 gives

$$
d^{\prime \prime}=\left(\frac{f_{\mathrm{e}}^{2}}{\left(l_{\mathrm{e} 2}^{\prime}+d^{\prime}\right)-g_{\mathrm{e}}}\right)+g_{\mathrm{e}}-l_{\mathrm{e} 2}^{\prime \prime}
$$

Substituting the preceding expressions into eq 1 gives

$$
\delta E_{\mathrm{cb}}=2\left\{\left(\frac{f_{\mathrm{e}}^{2}}{\left(l_{\mathrm{e} 2}^{\prime}+d^{\prime}\right)-g_{\mathrm{e}}}\right)+g_{\mathrm{e}}-l_{\mathrm{e} 2}^{\prime \prime}\right\} \frac{\theta^{\prime} E^{0}}{a_{\mathrm{e}}\left(1-G_{\mathrm{e}}\right) G_{\mathrm{e}}}
$$

For the instrument described here, in units of millimeters, $f_{\mathrm{e}}=338.58, l_{\mathrm{e} 2}^{\prime}=152.4, l_{\mathrm{e} 2}^{\prime \prime}=115.6, a_{\mathrm{e}}=381$, $g_{\mathrm{e}}=-205.08$, and $G_{\mathrm{e}}=-0.94713$. Figure 10 shows $\delta E_{\mathrm{cb}}$ plotted against $d^{\prime}$ for scattering angles of 0.001 , 0.003 , and $0.01 \mathrm{rad}$. The relationship is almost linear; it deviates only at larger values of $d^{\prime}$. Ideally the best operating point will be within a couple of millimeters 
of $d^{\prime}=0$; collision cells of greater length or those that are positioned incorrectly will degrade performance. For the experimental data measured for the scattering of $\mathrm{N}^{+}$the collisional broadening increases by 0.0034 $\mathrm{eV} \mathrm{mm} \mathrm{m}^{-1}$, which corresponds to a scattering angle of $0.00088 \mathrm{rad}$.

\section{Conclusion}

It has been shown that the energy resolution that can be obtained in collision-induced processes, in particular where fragmentation is not involved, can be increased by (1) reduction of the path length of the collision cell whilst target thickness is maintained and (2) correct positioning of the cell at the optical center of the instrument (or alternatively ensuring that the $Y$ focus lenses have moved the focal plane to an equivalent position). The main contribution to collisional broadening is a first-order effect. The ability to investigate collisional broadening processes is also feasible by precise measurement and analysis of the shapes of plots such as that shown in Figure 8c. Equations that describe collisional broadening to first order have been derived and adequately describe the phenomena that occur, which allows estimation of the scattering angle that arises from the collision-induced process.

\section{Acknowledgment}

$\mathrm{CL}$ and ACRW would like to thank the Engineering and Physical Science Research Council, for postgraduate studentships.

\section{References}

1. Cooks, R. G. Collision Spectroscopy; Plenum Press: New York, 1978.

2. McLafferty, F. W. Tandem Mass Spectrometry; Wiley: New York, 1983.

3. Hamdan, M.; Brenton, A. G. Springer Ser. Chem. Phys. 1991, $54,165$.

4. Barber, M.; Elliot, R. M. Proceedings of the 12th ASMS Conference on Mass Spectrometry and Allied Topics; Montreal, 1964.

5. Bruins, A. P.; Jennings, K. R; Evans, S. Int. J. Mass Spectrom. Ion Phys. 1978, 26, 395.

6. Morgan, R. P.; Beynon, J. H.; Bateman, R. H.; Green, B. N. Int. J. Mass Spectrom. Lon Plyys. 1978, 28, 171.

7. Beynon, J. H.; Brenton, A. G; Harris, F. M. Int. J. Mass Spectrom. Ion Phys. 1982, 45, 5.

8. Glish, G. L.; Todd, P. J. Anal. Chem. 1982, 54, 842.

9. Kobyashi, N. Springer Ser. Chem. Phys. 1991, 54, 123.

10. Enos, C. S.; Brenton, A. G., unpublished results.

11. Jonathan, P.; Hamdan, M.; Brenton, A. G.; Willet, G. D. Chem. Pliys. 1988, 119, 154.

12. Duckworth, H. E.; Barber, R. C.; Venkatasubramanian, V. S. Mass Spectrometry. Cambridge Monographs on Physics; Cambridge University Press, 1990. 